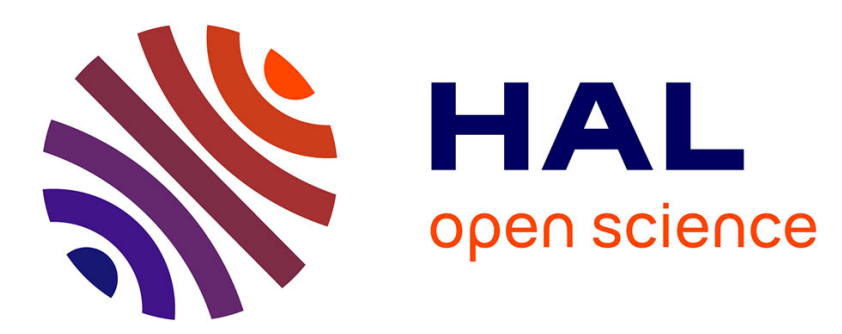

\title{
Application of Non-Linear Optical Methods to Plasma Diagnostics
}

\author{
U. Czarnetzki
}

\section{To cite this version:}

U. Czarnetzki. Application of Non-Linear Optical Methods to Plasma Diagnostics. Journal de Physique IV Proceedings, 1997, 07 (C4), pp.C4-175-C4-186. 10.1051/.jp4:1997413 . jpa-00255569

\section{HAL Id: jpa-00255569 https://hal.science/jpa-00255569}

Submitted on 1 Jan 1997

HAL is a multi-disciplinary open access archive for the deposit and dissemination of scientific research documents, whether they are published or not. The documents may come from teaching and research institutions in France or abroad, or from public or private research centers.
L'archive ouverte pluridisciplinaire HAL, est destinée au dépôt et à la diffusion de documents scientifiques de niveau recherche, publiés ou non, émanant des établissements d'enseignement et de recherche français ou étrangers, des laboratoires publics ou privés. 


\title{
Application of Non-Linear Optical Methods to Plasma Diagnostics
}

\section{U. Czarnetzki}

Institut für Laser- und Plasmaphysik, Universität GH Essen, 45117 Essen, Germany

\begin{abstract}
The application of non-linear optical methods nowadays allows the measurement of an increasing number of specific plasma quantities which were previously not amenable to diagnostics. Among those are state resolved measurements of molecular density distributions, sensitive measurement of light atomic radical densities, electric field measurements, and determination of quenching rates. In this paper an overview of the available techniques and trends is given. Some selected topics are discussed in more detail.
\end{abstract}

\section{INTRODUCTION}

Since the early days of plasma physics the emission of light from the discharge has always been a very valuable tool for the study of basic phenomena. This is still true today where emission spectroscopy is finding wide application not only in research but also in process monitoring in industrial applications $[1,2,3]$. The sensitivity of the spectra to changes of the discharge conditions and the relatively modest experimental requirements have contributed to this development. However, to obtain an unambiguous picture of the physical conditions that lead to the measured spectra turns out to be often very difficult and one has to be very careful when drawing conclusions. This is partly due to the complexity of the problem and partly to the lack of reliable atomic and molecular cross-sections [4].

Laser spectroscopy often allows a more direct interpretation of the measured data and in addition can be a more sensitive probe with, at the same time, better spatial and temporal resolution. Although for most laser techniques the experimental requirements are more demanding, many methods have become standard in plasma diagnostics. Examples are the determination of electron and ion distributions and densities by Thomson scattering, laser induced fluorescence spectroscopy for the measurement of neutral particle densities and ion drift velocities, and IR-absorption spectroscopy by laser diodes for the measurement of molecular populations in the ground states [5-10]. These techniques have in common that the generated signal is proportional to the laser intensity and to the number of particles involved as long as saturation is avoided. Therefore they are called linear laser spectroscopy. For practical applications it might also be important that only a single laser system is required. Although these techniques are very powerful diagnostic tools, there are certain technical and basic physical limitations, e.g. available laser wavelengths or powers, selection rules for dipole transitions or absorption of gases in the VUV.

Nonlinear laser spectroscopy techniques allow some of these constraints to be overcome. In addition to serving as a diagnostic tool nonlinear optical techniques can also be applied to the generation of radiation necessary for linear as well as nonlinear spectroscopy. It is the aim of this paper to give an overview of the available techniques and to discuss briefly some examples of their applications to plasma diagnostics. However, in this frame it is not the intention to give a complete review of experiments and applications of nonlinear optics in plasma physics or to discuss all variants and aspects of a particular technique. 
In the following a brief introduction to nonlinear spectroscopy will be given that leads to the classification of the basic phenomena into certain groups. For every group examples of application to plasma diagnostics will then be discussed in more detail.

\section{SOME BASIC PRINCIPLES OF NONLINEAR LASER SPECTROSCOPY IN PLASMAS}

Nonlinear optical phenomena in the plasma itself (free electrons), like e.g. harmonic generation, parametric amplification, and phase conjugation, have been the subject of intense research especially in connection with laser induced plasmas and laser fusion [11-16]. However, in the framework of this paper nonlinear phenomena are defined to be connected to the interaction of the laser beams with neutral particles, i.e. with bound electrons. It is further assumed that no strong gradients are present and that the medium can be considered as isotropic. This assumption is justified in most cases of low as well as high temperature plasma applications.

The interaction of radiation with matter is described by Maxwell's equations together with the susceptibility tensor [17-18]. The susceptibility times the electric fields acts as the source term in the wave equations. This means that the electric fields of the laser beams are inducing a polarization in the medium and that this polarization generates a wave at the sum or difference of the frequencies of the incoming waves. As a consequence of the isotropy only nonlinear optical phenomena of third or higher odd order in the susceptibility are possible. Phenomena well known from optical crystals like frequency doubling are not possible in a plasma. With increasing order of the nonlinear effect the interaction efficiency is drastically reduced and therefore only third order effects or combinations of third and first order techniques have found application in plasma spectroscopy so far.

In general four groups of nonlinear phenomena can be distinguished. As in linear spectroscopy the real and imaginary part of the susceptibility describe the refractive index and the absorption of radiation, respectively. Although nonlinear in the intensity they are still linear with respect to the particle density. The most important example is two-photon absorption. Nonlinear dispersion as an isolated phenomenon is of no importance for plasma diagnostics. While absorption and dispersion are still similar to what is known from linear spectroscopy the following group has no such counterparts and a more detailed introduction will be given.

The third group comprises phenomena based on the square of the absolute value of the susceptibility. These spectroscopic techniques are called four-wave mixing and they depend quadratically on the particle density. Probably the best known example is coherent anti-Stokes Raman scattering (CARS). No energy is dissipated in the medium but it acts like a "catalyst" for the generation of radiation, i.e. the sum of the photon energy of the incoming laser beams and the generated signal wave is conserved. Therefore the frequency of the signal wave can be only the sum or difference of the generating laser frequencies.

The generated signal wave is highly directed with a low divergence like a laser beam and makes application especially favorable in cases of high radiation background. The reason for this directionality is the conservation of momentum. Since the atoms or molecules do not absorb photons the momentum of the photons has also to be conserved. In other words the sum of the k-vectors of the four waves involved has to be zero. Since the k-vector times the interaction length gives the phase of a wave this condition is called phase-matching. As a consequence propagation of the signal wave is only possible in a certain direction defined by the directions of the laser beams. Dispersion in the medium can change the value of the $\mathrm{k}$-vectors and therefore for given frequencies this can restrict propagation to certain directions. This is especially pronounced in the VUV and close to atomic or molecular one-photon resonances where dispersion is strong and is an important effect for the generation of VUV radiation by frequency mixing or high-order anti-Stokes Raman scattering.

However, atomic and molecular resonances also greatly enhance the efficiency of four-wave mixing. For example the CARS technique is taking advantage of two-photon resonances to selectively probe vibrational and rotational levels. Since four-wave mixing generates radiation, it has found application as a direct diagnostic technique as well as a method for the efficient conversion of tunable laser radiation to frequencies that can not be generated otherwise. This radiation can then be again applied to linear or non-linear plasma spectroscopy. 
As a fourth group one may consider applications that are a combination of linear and nonlinear techniques like e.g. laser induced fluorescence among excited states after a previous two-photon excitation of the atom.

Nonlinear spectroscopic techniques in plasma diagnostics are therefore either based on nonlinear absorption, four-wave mixing, or a combination of linear and nonlinear methods. Examples for these groups will be given in the following. In addition to be a spectroscopic technique, four-wave mixing is also applied to the generation of VUV radiation for linear spectroscopy.

\section{APPLICATIONS TO THE GENERATION OF RADIATION FOR LNEAR SPECTROSCOPY}

Without going into the details of the nonlinear processes two examples from high and low temperature plasmas should be mentioned. Here the radiation is generated by nonlinear optics but the spectroscopy is still linear. The selected examples show applications of the two most common methods for generation of laser radiation in the vacuum ultraviolet (VUV).

For the detection of atomic hydrogen in the boundary layers of the tokamak experiment TEXTOR Bogen and Mertens developed a frequency tripling cell for the generation of coherent radiation at Lyman- $\alpha[19,20]$. The frequency tripling cell filled with a mixture of krypton and argon converts radiation at $364.68 \mathrm{~nm}$ coming from a dye laser down to the third harmonic at $121.56 \mathrm{~nm}$. The generated radiation is used for the measurement of atomic hydrogen densities by laser induced fluorescence spectroscopy. With particles at room temperature densities of the order of less than $10^{8} \mathrm{~cm}^{-3}$ could be detected.

The population of the vibrational and rotational states of molecular hydrogen and deuterium in the electronic ground state is of great importance for the understanding of the formation of negative ions in magnetic multi-cusp sources. These populations can be measured with high sensitivity by absorption spectroscopy. However, tunable radiation in the VUV in the spectral range between $120 \mathrm{~nm}$ and $140 \mathrm{~nm}$ is required. This radiation has been generated in an experiment performed by Wagner and Döbele by stimulated anti-Stokes Raman scattering [21]. Population of vibrational levels between $v=2$ and $v=6$ could be investigated. In this case a cell filled with molecular hydrogen converts the pump radiation coming from a dye laser to a series of anti-Stokes orders spectrally displaced from the pump frequency by an integer number of the Raman-transition frequency of $4155 \mathrm{~cm}^{-1}$. Up to 15 anti-Stokes orders have been reported in the literature $[22,23]$

Recently the generation of very high anti-Stokes orders at short VUV wavelengths between $120 \mathrm{~nm}$ and $140 \mathrm{~nm}$ has been greatly improved by the combination of two Raman-cells (Fig. 1) [24,25]. Tunable dye-laser radiation around $370 \mathrm{~nm}$ is first passing through a high pressure Raman-cell filled with hydrogen. There a small amount of radiation at the wavelength of the first Stokes-order is generated. The

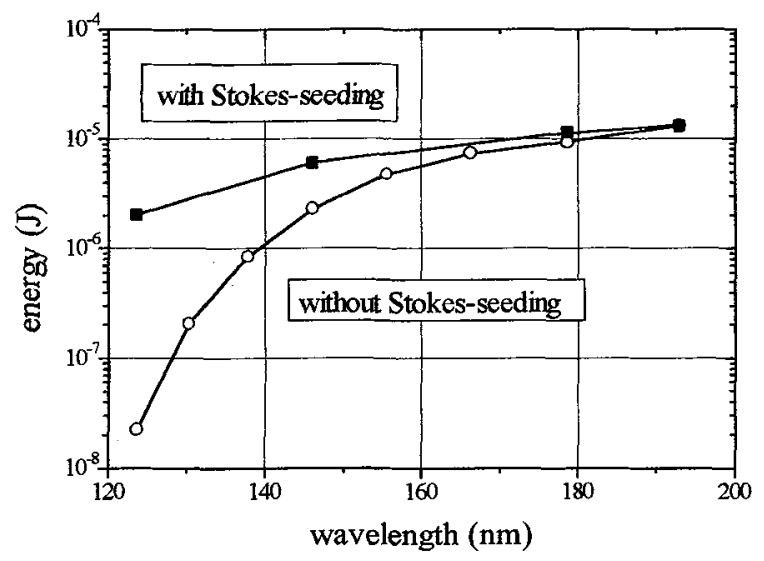

Figure 1: Energies of various anti-Stokes orders with and without Stokes-seeding. 
combined beams are then focused into a subsequent Raman-cell at normal pressure where the high antiStokes orders are generated. This method is called "Stokes-seeding".

For the four-wave mixing process that generates high anti-Stokes orders the presence of the first Stokes component is essential. With a single Raman-cell the first Stokes-component builds up from quantum noise. Therefore the entire process can be improved essentially if a small portion of Stokes radiation is already present initially. The spectral region in the VUV is especially interesting for laser induced fluorescence spectroscopy in atomic hydrogen $(121 \mathrm{~nm})$ and oxygen $(130 \mathrm{~nm})$.

\section{APPLICATIONS BASED ON NONLINEAR ABSORPTION}

Two-photon absorption laser induced fluorescence spectroscopy (TALIF) is one of the most widely applied methods of nonlinear optics in plasma diagnostics [26,27,28]. Although already predicted by Maria Göppert-Mayer in 1931 [29] it was not until the development of the laser that this phenomenon could be observed in the optical region. The reason for this is obvious when one looks at the required intensities. While in one-photon laser induced fluorescence spectroscopy usually intensities of the order of 100 $\mathrm{Wcm}^{-2}$ are sufficient for the saturation of a transition, two-photon excitation requires intensities of typically $10^{9} \mathrm{Wcm}^{-2}$ to reach a similar population in the excited state [30]. However, modern pulsed laser systems can easily deliver such intensities when the laser beam is focused. For instance $1 \mathrm{~mJ}$ in a $10 \mathrm{~ns}$ pulse focused to a spot diameter of $0.1 \mathrm{~mm}$ gives an intensity of $10^{9} \mathrm{Wcm}^{-2}$. Although the required intensities can easily be reached, problems can arise in complex molecular media where one has to avoid photo-dissociation. Excitation wavelengths are usually in the UV and therefore photo-dissociation can be very efficient $[31,32]$. Another limiting factor is photo-ionisation by a third photon. This gives rise to saturation of the excitation efficiency at typically a few percent of the ground state population [30]. As long as saturation can be neglected the number of excited atoms scales with the square of the laser intensity.

But there are some more differences to the one-photon case. Because of the selection rules twophoton excitation and one-photon excitation are exclusive to each other: Two states that can be coupled by a one-photon dipole transition can not be coupled by a two-photon transition and vice versa. The emission of fluorescence light from the excited state is always at a wavelength different from the exciting radiation if no intermediate resonance is involved, which is usually not the case. Since the transition energy is twice that of a single photon large interatomic energy gaps can be spanned without the need of having radiation in the VUV - a great advantage especially for light radicals like e.g. $\mathrm{H}, \mathrm{O}$, or $\mathrm{N}$.

A further improvement of two-photon excitation can be made by Doppler-free excitation by two counterpropagating beams. Atoms can absorb one photon from one beam and another from the beam traveling in the opposite direction. If the atom is moving at a certain velocity it will see one beam Doppler-shifted to the red and the other beam shifted to the blue by exactly the same amount. Therefore in the sum the Doppler-shift cancels out and independent of their individual velocities all atoms are equally excited. This Doppler-free technique offers high excitation efficiency and high spectral resolution at the same time [26,33]. However, it requires a laser bandwidth much smaller than the Doppler-width, a requirement often difficult to match with pulsed lasers.

Another problem connected with TALIF is the calibration in order to measure not only relative but absolute densities. A method often applied is the generation of the species under investigation at a known amount in a so called "flow-tube reactor" $[34,35]$. The reactor can usually be externally attached to the discharge chamber. In a microwave discharge the atomic species are generated by dissociation. The atoms are then carried through a tube to the laser focus in the experimental chamber. Therefore the excitation and detection set-up remains unchanged. By adding a certain gas at a known rate the atomic species is then destroyed in a chemical titration reaction. From the slope of the decrease of the fluorescence signal as a function of the flow of the added titration gas together with the total flow and pressure one can deduce the total particle density at the beginning of the titration. This finally gives the calibration constant. 
Tab. 1: Two-photon excitation and fluorescence emission wavelengths for some atoms.

$\begin{array}{ccc}\text { Atom } & \begin{array}{c}\text { 2-photon transition } \\ \text { wavelength }(\mathrm{nm})\end{array} & \begin{array}{c}\text { Fluorescence wave- } \\ \text { length }(\mathrm{nm})\end{array} \\ \mathrm{H} & 205 & 656 \\ \mathrm{~N} & 211 & 871 \\ \mathrm{C} & 287 & 166 \\ \mathrm{O} & 225 & 845 \\ \mathrm{Cl} & 210 & 904\end{array}$

A list of two-photon excitation wavelengths for various light atoms is given in table 1. Atomic hydrogen is an interesting example since it allows various alternative excitation schemes as shown in Fig. 2 [30]. An example of a density profile measured by Doppler-free two-photon excitation at $205 \mathrm{~nm}$ in a capacitively coupled RF-discharge in the GEC-reference cell is shown in Fig. 3. Comparison is made with emission spectroscopy at Balmer- $\alpha$. Due to the gain in sensitivity by the Doppler-free technique an unfocussed laser beam of $5 \mathrm{~mm}$ diameter is used in this experiment and detection is by a CCD camera perpendicular to the laser beam. This allows instantaneous spatial resolution along the radial direction of the discharge [36].

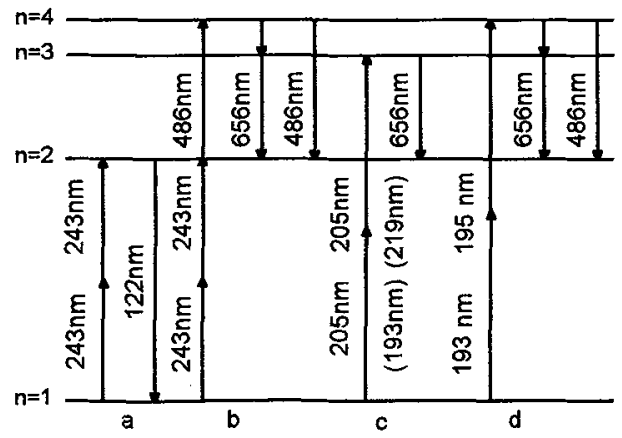

Figure 2: Various alternative two-photon excitation schemes in atomic hydrogen.

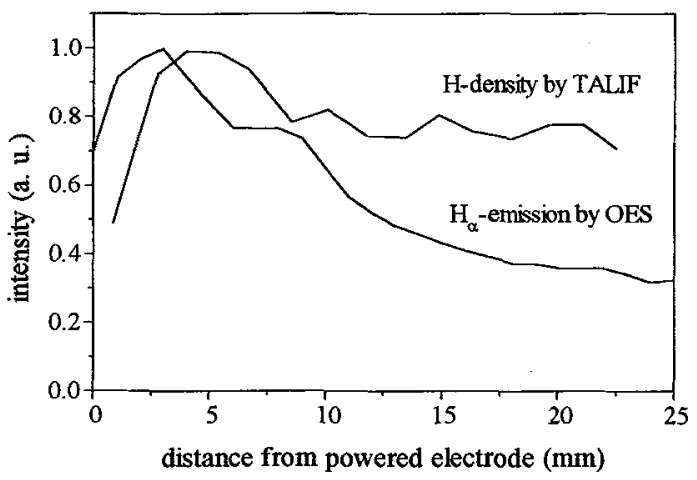

Figure 3 :Axial density distribution of atomic hydrogen atoms measured by two-photon absorption laser induced fluorescence spectroscopy (TALIF) and intensity distribution of plasma induced optical emission at $\mathrm{H}_{\alpha}(\mathrm{OES})$ in a hydrogen capacitively coupled RF-discharge in the GEC-reference cell. 


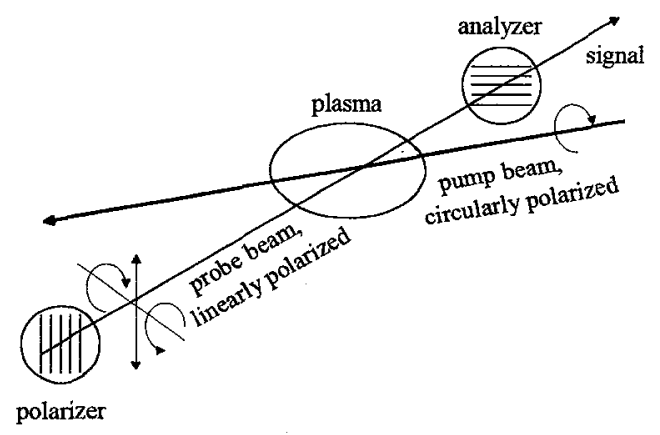

Figure 4: Scheme for two-photon polarization spectroscopy.

A problem connected with spectroscopy in general is quenching of the upper state by collisions with other neutrals or ions and electrons $[37,38]$. Although less sensitive than LIF, absorption spectroscopy avoids this problem and probes directly the ground state population. Grützmacher et al. [39] have developed a nonlinear Doppler-free absorption technique called "two-photon polarization spectroscopy" that depends on both, the squares of the real and the imaginary part of the nonlinear susceptibility and takes advantage of the selection rules for two-photon transitions.

Two laser beams are counter-propagating and intercept over a certain region within the plasma (Fig. 4). One beam is circularly polarized, e.g. clock wise, this is the pump beam, and the other beam is linearly polarized, e.g. in the x-direction, this is the probe beam. Both beams have slightly different wavelengths, $248.5 \mathrm{~nm}$ and $237.8 \mathrm{~nm}$, so that only the combination of the two is in resonance with the twophoton transition between the $1 \mathrm{~s}$ and $2 \mathrm{~s}$ states in atomic hydrogen. The linearly polarized probe beam can be considered as a superposition of two circularly beams with opposite helicity. Since the angular momentum in the atom is not changing in the absorption process only the clock wise circularly polarized part of the probe beam will be absorbed. This gives rise to the appearance of a component in the probe beam that is polarized perpendicular to the initial direction. This component is measured behind a polarizer and scales with the squares of density, interaction length, and nonlinear susceptibility. Actually a more rigorous treatment shows that the generation of the perpendicular component can also be understood as a four-wave mixing process.

Since the effect is very small this technique can be applied only at relatively high densities in the excess of $10^{13} \mathrm{~cm}^{-3}$. However, this is also the region where quenching usually has an effect that this technique avoids in an elegant way. The authors have demonstrated their method in an electrical arc plasma at $10 \mathrm{kPa}$.

The knowledge of quenching rates is also of great importance for emission spectroscopy: A widely applied emission technique, especially in thin film diamond deposition, is actinometry with argon. In order to obtain the quenching rate of the argon $750.4 \mathrm{~nm}$ line time resolved TALIF was applied [40]. The necessary radiation for two-photon excitation at $184 \mathrm{~nm}$ was generated by stimulated anti-Stokes Raman scattering in hydrogen. Starting with dye-laser radiation at $396 \mathrm{~nm}$ the 7 th anti-Stokes component is at the desired wavelength. The quenching rates are deduced from the change in the decay of the fluorescence light with increasing gas pressure. An example with acetylene is shown in Fig. 5. Here nonlinear optical methods have been applied to the generation of the radiation as well as for the diagnostic method itself.

\section{APPLICATIONS BASED ON FOUR-WAVE MIXING}

Probably the most prominent of the four-wave mixing techniques is coherent anti-Stokes Raman scattering (CARS) [41-46]. It is a technique that can measure the rotational and vibrational population of molecules in the ground state, e.g. $\mathrm{H}_{2}, \mathrm{~N}_{2}, \mathrm{CO}_{2}, \mathrm{C}_{2} \mathrm{H}_{2}, \mathrm{CH}_{4}$. Due to the selection rules for optical transition in 


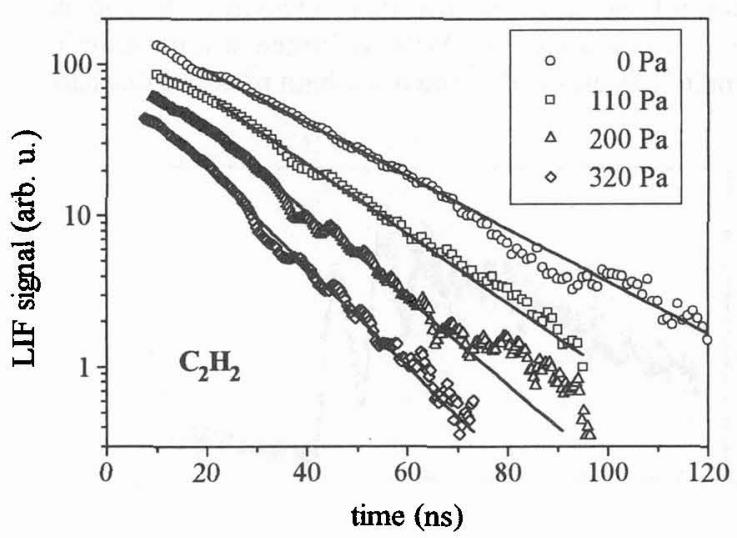

Figure 5: Decay of argon $750.4 \mathrm{~nm}$ fluorescence emission after two-photon excitation at various pressures of acetylene. The argon pressure is kept constant at $50 \mathrm{~Pa}$.

molecules Raman and infra-red absorption transitions are exclusive. Therefore the two techniques complement one another.

Like in all four-wave mixing techniques three incoming laser beams are generating a fourth beam that is emitted into a direction that is defined by the phase matching condition and the direction of the incoming beams. The amplitude of the signal is proportional to the square of the density, the interaction length, and the nonlinear susceptibility and depends linearly on the intensities of the three incoming beams. An energy level diagram for CARS is shown in Fig. 6. A pump beam at $\omega_{\mathrm{p}}$ and a Stokes-beam at $\omega_{s}$ are at a two-photon resonance between the initial state (i) and a state (s). Both states are rovibronic states of the electronic ground state of the molecule. The third beam, which is also at $\omega_{\mathrm{p}}$ then generates an anti-Stokes wave at $\omega_{\mathrm{as}}$ by a two-photon resonant transition back to the initial state. If the laser at $\omega_{\mathrm{s}}$ is detuned off the resonance between the states (i) and (s) the anti-Stokes generation will terminate. Therefore by tuning the Stokes frequency the various molecular resonances can be probed and the population in these states can be measured. From these data rotational and vibrational temperatures can be directly derived. The selectivity and high spectral resolution and the directionality of the signal beam are great advantages of the CARS method.
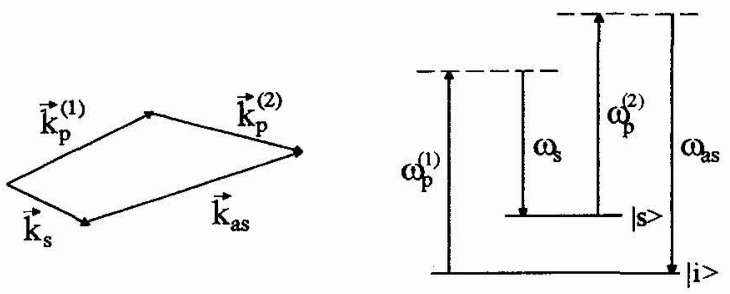

Figure 6: Simplified scheme for coherent anti-Stokes Raman scattering (CARS).

In order to measure absolute densities a calibration procedure is necessary. Often a reference cell with a known concentration of the species to be measured is used. It should be noted that the analysis of the CARS spectra usually requires a detailed knowledge of the molecular constants and processes and is carried out by comparing them with theoretical simulations. An example of a comparison between a measured and a calculated CARS spectrum in acetylene is given in Fig. 7 [47]. The minimal detectable particle density per state is typically of the order of $10^{11} \mathrm{~cm}^{-3}$ or above.

A very interesting variant of the CARS technique has been presented by Ochkin et al [48]. Actually the four waves present in the CARS process must not necessarily all be waves. If one replaces the second pump beam at $\omega_{\mathrm{p}}$ by a static electric field there will still be a generated signal wave, now with a frequency corresponding to the energy gap between the two levels involved. The amplitude of this signal wave is 
then proportional to the square of the static electric field. Ochkin et al. applied this novel technique of electric field measurement to a corona discharge. With hydrogen at atmospheric pressure their sensitivity was about $25 \mathrm{~V} / \mathrm{cm}$. This technique is especially suited for high pressure discharges.

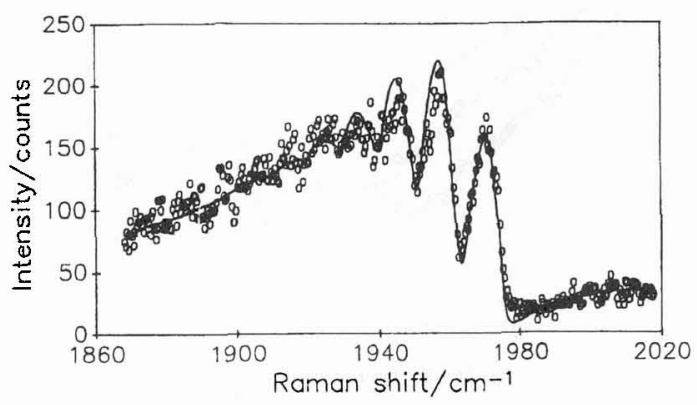

Figure 7: Comparison of measured and calculated CARS-spectrum in acetylene.

Another recent development in CARS diagnostics in plasmas is the so called resonant CARS (RECARS) [41,49]. The difference between the two methods is the following: As shown in Fig. 6 two twophoton steps are involved in the CARS process. For these two-photon transitions the excited electronic states of the molecule serve as the intermediate states and they are all far from resonance in the ordinary CARS process. However, if one or both of the pump lasers get in resonance with these states the efficiency of the CARS process is greatly enhanced. RECARS has been applied to the detection of e.g. $\mathrm{CH}$ in an $\mathrm{Ar} / \mathrm{CH}_{4}$ microwave discharge.

A double-resonant four-wave mixing scheme has been proposed for the detection of atomic hydrogen [50](Fig. 8). Here two beams at $205 \mathrm{~nm}$ are in resonance with the two-photon transition between the ground state and $n=3$. The third beam is close to the resonance at Balmer- $\alpha$ and generates a signal wave close to Lyman- $\alpha$. Like in the CARS-scheme this signal wave is highly directed and allows the efficient suppression of background radiation by spatial filtering. In a first experiment this novel technique was tested on atomic hydrogen generated by dissociation at a hot filament [51]. At elevated energies of the visible laser with the laser tuned on resonance the process gets saturated. In the saturated case the dependence of the signal on the particle density changes from quadratic to linear. This is shown in Fig. 9. The particle density was about $10^{13} \mathrm{~cm}^{-3}$. Theoretical calculations predict a possible detection threshold of the order of $10^{9} \mathrm{~cm}^{-3}$.

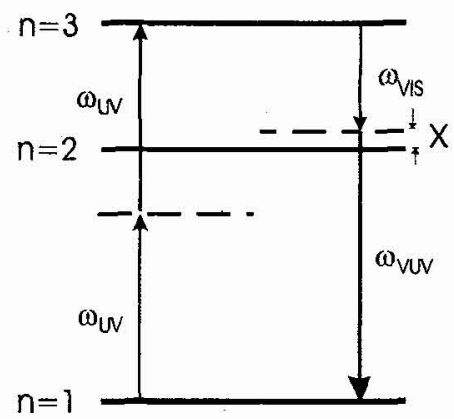

Figure 8: Scheme for double-resonant four-wave mixing (DORFWM).

So far the four waves involved had different frequencies. However, four-wave mixing is also possible when all four waves have identical frequencies. This is called degenerate four-wave mixing (DFWM) $[52,53]$. Two different schemes can be distinguished: DFWM with a two-photon resonance and with a one-photon resonance. Both schemes can be understood in terms of transient spatial gratings. Two strong pump beams form a spatial interference pattern where the grating constant depends on the angle 


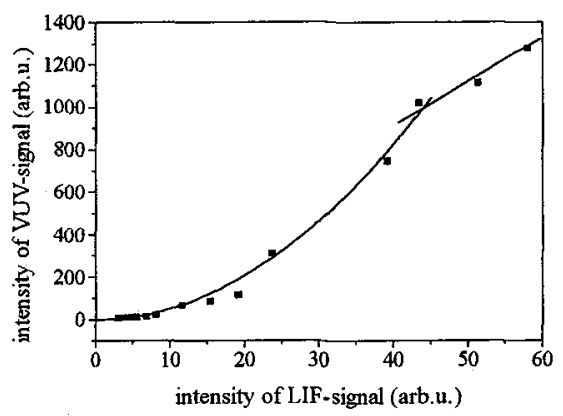

Figure 9. Saturation behavior of the DORFWM-signal as a function of the particle density.

between the two beams. This interference pattern is then reflected in the spatial excitation distribution that forms a similar grating. A probe beam is then scattered at this grating and a signal beam is generated. With counterpropagating beams this scheme is Doppler-free and offers a high spectral resolution. Since DFWM does not depend on the emission of fluorescence light the effect of quenching is greatly reduced. In the case of DFWM with one-photon resonances the signal scales with an exponent between 8 and 2 of the transition dipole moment, depending on the degree of saturation [54]. This makes absolute concentration measurements difficult and also often limits the application to relatively high densities.

DFWM has been applied to the detection of molecular and atomic species like e.g. $\mathrm{H}_{2}, \mathrm{CH}, \mathrm{OH}$, $\mathrm{NH}, \mathrm{NO}_{2}, \mathrm{C}_{2}, \mathrm{He}, \mathrm{Hg}, \mathrm{Na}$ [55-57]. An example for the application of the one-photon scheme are electric field measurements in helium $[58,59]$. Electric fields of the order of $500 \mathrm{~V} / \mathrm{cm}$ have been measured in the sheath of a glow discharge. The experimental set-up is shown in Fig. 10. The grating is formed by the forward beam together with the reflected beam from the mirror. A small fraction of the probe beam is scattered backwards at the grating and detected by a photomultiplier. By tuning the frequency of the laser the Stark-splitting of Rydberg-states is measured and from this splitting the electric field is deduced.

A two-color six-wave mixing scheme, which is actually a process of 5 th order in the nonlinear susceptibility, has been demonstrated by Gray et al. [60] for the detection of atomic hydrogen. They report a detection threshold at about $10^{13} \mathrm{~cm}^{-3}$. The experiment was performed in a flame, however, it might also find application in plasma diagnostics in the future.

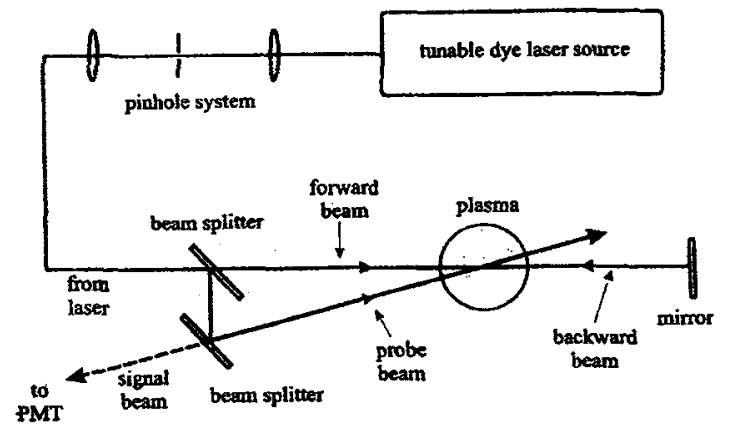

Figure 10: Experimental setup for electric field measurements in helium by degenerate four-wave mixing (DFWM).

\section{COMBINED TECHNIQUES}

In this chapter two examples will be given where nonlinear and linear spectroscopic methods have been combined to measure electric field distributions in atomic hydrogen. Electric fields can be measured by probing the Stark splitting of Rydberg states where the splitting scales with the principle quantum 
number like $n(n-1)$. Since atomic hydrogen can not be excited directly from the ground state due to the large excitation energy a multi-photon scheme has to be applied.

Booth et al. [61] therefore used a two-photon excitation at $243 \mathrm{~nm}$ for excitation to the $n=2$ level. With a third photon at $410 \mathrm{~nm}$ they then further exited the atom to the $n=6$ state. The laser at $243 \mathrm{~nm}$ was fixed on resonance while the laser at $410 \mathrm{~nm}$ was tuned over the Stark split $n=6$ state. Fluorescence light after collisional population transfer to $n=3$ was observed at the Balmer- $\alpha$ transition. Electric fields were measured in the cathodic layer of a $30 \mathrm{kHz}$ discharge in hydrogen with a sensitivity as low as 20 $\mathrm{V} / \mathrm{cm}$.

With increasing quantum number of the Rydberg state the electric field sensitivity is increasing, however, at the same time the signal amplitude is drastically decreasing. This is due to the long life time of the Rydberg states and the unfavorable branching ratio. Recently we developed a novel technique in hydrogen that can overcome these limitations [62]. In our scheme we excite the atom in a two-photon step at $205 \mathrm{~nm}$ to the $n=3$ state (Fig. 11). This excitation is Doppler-free and the laser beams are unfocused, therefore allowing instantaneous spatial resolution. Fluorescence light at $656 \mathrm{~nm}$ is observed. A second laser is now tuned to the resonance between $n=3$ and a Rydberg state. Whenever this laser is in resonance with one of the Stark-split components of this state, population is transfered from $n=3$ to the Rydberg state. This is noticed in a decrease of the fluorescence signal at Balmer- $\alpha$. Since this does not depend on the lifetime of the Rydberg state or on the branching ratio or on the collisional transfer almost any Rydberg state can be probed. The limiting factor for the Rydberg-state quantum number is the energy necessary to transfer a noticeable amount of population from $n=3$ to the Rydberg state. This energy is increasing with increasing principle quantum number. Nevertheless, high Rydberg-states up $n=26$ or even higher should be accessible. This could also prove to be an alternative method for the determination of electron densities.

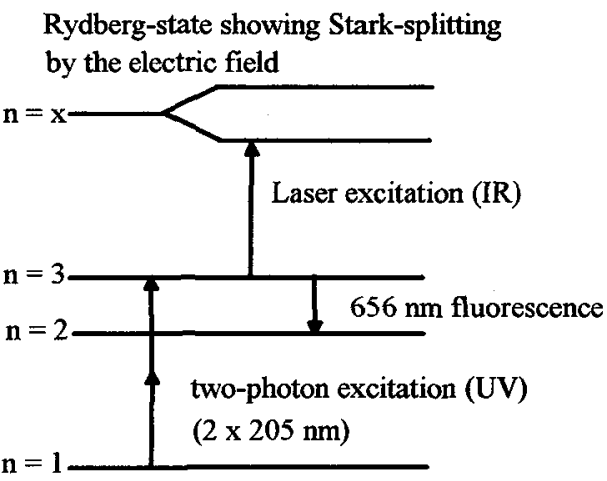

Figure 11: Scheme for optical double-resonance spectroscopy in hydrogen.

In a first experiment up to $n=19$ could be probed in the sheath of an RF-discharge in hydrogen. An example at $n=16$ and about $1060 \mathrm{~V} / \mathrm{cm}$ is shown in Fig. 12. At high field strengths the Stark-

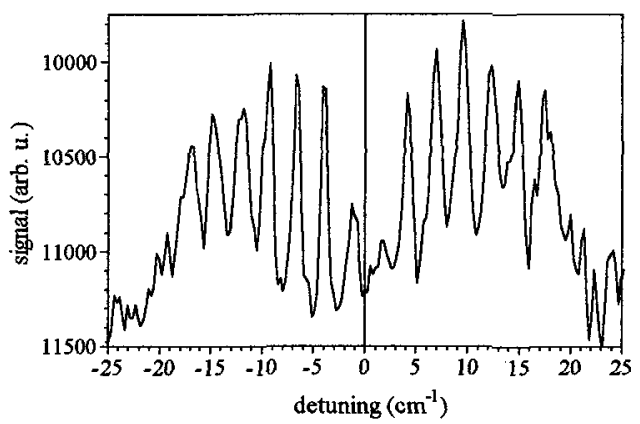

Figure 12: Stark-splitting of $\mathrm{H}$-atom, $\mathrm{n}=16$ at $1060 \mathrm{~V} / \mathrm{cm}$. 
components are all well resolved. However, for the determination of the electric field strength it is sufficient to measure the width of the spectrum which is possible also at much lower values. Therefore this method seems to be a very promising candidate for the measurement of very low electric fields of the order of $10 \mathrm{~V} / \mathrm{cm}$ or less in e.g. the positive column of glow discharges or in inductively coupled RF-discharges.

\section{SUMMARY}

Nonlinear laser spectroscopy techniques have proved to be a very powerful tool for plasma diagnostics. They are not a substitute for, but complementary to conventional techniques. Recent improvements in laser systems and detector techniques have opened new fields of application. Novel techniques have been developed and established techniques from other fields of laser spectroscopy have found new applications to problems in plasma physics. This has opened windows to areas that have been previously inaccessible. In addition knowledge gained from nonlinear laser spectroscopy can also turn out to be very valuable for the further development and improvement of more conventional diagnostic techniques.

\section{References}

[1] Hans R. Griem, Plasma Spectroscopy (McGraw-Hill, 1964)

[2] H.-J. Kunze: In J. M. Proud and L.H. Luessen, eds.: Radiative Processes in Discharge Plasmas (Plenum Press, New York, 1986)

[3] Z. Quing, D.K. Otorbaev, G.J.H. Brussaard, M.C.M. van de Sanden, and D.C. Schram, J. Appl. Phys. 80, 1312 (1996)

[4] V. Schulz-von der Gathen and H.F. Döbele, Plasma Chemistry and Plasma Processing 16, 461 (1996)

[5] H.-J. Wesseling and B. Kronast, J. Phys. D. 29, 1035 (1996)

[6] K. Muraoka and M. Maeda, Plasma Phys. and Contr. Fus. 35/6, 633-656 (1993)

[7] E.A. Den Hartog, H. Persing, and R.C. Woods, Appl. Phys. Lett. 57, 661 (1990)

[8] K. Niemax, H. Groll, and C. Schnürer-Patschan, Spectrochimica Acta Rev. 15, 349 (1993)

[9] M. Ikeda, K. Aiso, M. Hori, and T. Goto, Jpn. J. Appl. Phys. 34, 3273 (1995)

[10] G. Bachet, L. Chérigier, M. Carrère, and F. Doveil, Phys. Fluids B 5, 3097 (1993)

[11] A. Lal and C. Joshi, J. Opt. Soc. Am. B 8, 2148, (1991)

[12] D. Steel and J. Lam, Opt. Lett. 4, 363, (1979)

[13] J. Federici and D.K. Mansfield, J. Opt. Soc. Am. B 3, 1586 (1986)

[14] Y. Kitagawa, R. Savage, and C. Joshi, Phys. Rev. Lett. 62, 151, (1989)

[15] I. Nebenzahl, A. Ron, and N. Rostoker, Phys. Rev. Lett. 60, 1030 (1988)

[16] M. V. Goldman and E. A. Williams, Phys. Fluids B 3, 751, (1991)

[17] Y. R. Shen, The Principles of Nonlinear Optics (John Wiley \& Sons, 1984)

[18] A. Yariv, Quantum Electronics (John Wiley \& Sons, 1975)

[19] Ph. Mertens and P. Bogen, J. Nucl. Mat. 128 \& 129, 551 (1984)

[20] Ph. Mertens and P. Bogen, Appl. Phys. A 43, 197 (1987)

[21] D. Wagner and H.F. Döbele, to be published

[22] V: Schultz-von der Gathen, T. Bornemann, V. Kornas, and H.F. Döbele, IEEE J. Quant. Electr. 26 (4), (1990)

[23] U. Czarnetzki, H.F. Döbele, M. Hörl, M. Röwekamp, B. Reimann, V. Schultz von der Gathen, J. Nucl. Mat 86

[24] M. Spaan, A. Goehlich, V. Schultz-von der Gathen, and H.F. Döbele, Appl. Opt. 33, 3865 (1994)

[25] A. Wolf, M. Thomson, U. Czarnetzki, and H.F. Döbele, to be published

[26] K. Grützmacher, M.I. de la Rosa, A. Steiger, W. Bohmeyer, H. Grote and E. Parsch, P7.4 in Verhandl. DPG 30, 176 (1995) 
[27] A. D. Tserepi and T.A. Miller, J. Appl. Phys. 77, 505 (1995)

[28] J.E.M. Goldsmith, J. Opt. Soc. Am. B 11, 1979 (1989)

[29] M. Göppert-Mayer, Ann. Phys. 9, 273 (1931)

[30] U. Czarnetzki, K. Miyazaki, T. Kajiwara, and K. Muraoka, J. Opt. Soc. Am. B 11, 2155 (1994)

[31] K. Miyazaki,T. Kajiwara, K. Uchino, and K. Muraoka, J. Vac. Sci. Technol. A 14, 125 (1996)

[32] J.E.M. Goldsmith, Appl. Optics 28 (6), (1989)

[33] W. Demtröder, Laser Spectroscopy (Springer-Verlag Berlin Heidelberg New York, 1981)

[34] D. W. Setser, Reactive Intermediates in the Gas Phase (Academic, New York, 1979)

[35] U. Meier, K. Kohse-Höinghaus, L. Schäfer, and C.-P. Klages, Apl. Opt. 29, 4993 (1990)

[36] L. Cherigier, D. Luggenhölscher, U. Czarnetzki, and H. F. Döbele, to be published

[37] J. Bittner, K. Kohse-Höinghaus, U. Meier, and Th. Just, Chem. Phys. Lett. 143, 571 (1988)

[38] B. L. Preppernau, K. Pearce, A. Tserepi, E. Wurzberg, and T. A. Miller, Chem. Phys. 196, 371 (1995)

[39] R. Dux, K. Grützmacher, M. I. de la Rosa, B. Wende, Phys. Rev. E 51, 1416 (1995)

[40] A. Francis, N. Sadeghi, U. Czarnetzki, and H.F. Döbele, submitted to Appl. Phys. Lett. (1997)

[41] S. A. J. Druet and J.-P. Taran, Prog. Quant. Electr. 7, 1-77 (1981)

[42] T. Doerk, J. Ehlbeck, P. Jauernick, J. Stanco, J. Uhlenbusch, T. Wottka, J. Phys. D: Appl. Phys. 26, 1015 (1993)

[43] D. A. Greenhalgh, in, Advances in Nonlinear Spectroscopy, R.J.H. Clark, R.E. Hester, eds., Wiley, London (1988)

[44] D. Maker and R.W. Terhune, Phys. Rev. 137 (1965) A 801- A818

[45] J.W. Nibler, G.V. Knighton, in, Raman Spectroscopy of Gases and Liquids, Ed. A. Weber, Springer Verlag, Berlin 1979

[46] M.D. Levenson, Introduction to nonlinear Laser Spectroscopy, Academic Press, New York 1982

[47] V. Kornas, A. Roth, H.F. Döbele, and G. Proß, Plasma Chemistry and Plasma Processing 15, 71 (1995)

[48] O.A. Evsin, E.B. Kupuyanova, V.N. Ochkin, S.Y. Savinov, and S. N. Tsakhai, Quantum Electr. 22, 295 (1995)

[49] T. Doerk, P. Jauernik, S. Hädrich, B. Pfelzer, and J. Uhlenbusch, Opt. Commun. 118, 637 (1995)

[50] U. Czarnetzki and H.F. Döbele, Rev. Sci. Instrum. 66, 587 (1995)

[51] M. Thomson, U. Czarnetzki, and H.F. Döbele, to be published

[52] H.J. Eichler, P. Günter, and D.W. Pohl, Laser Induced Dynamic Gratings, (Springer, Berlin 1986)

[53] R.A. Fisher, ed., Optical Phase Conjugation (Academic Press, New York 1993)

[54] R.L. Farrow, D. Rakestaw, and T. Dreier, J. Opt. Soc. Am. B, 9 (19), 1770 (1992)

[55] G. Meijer and D.W. Chandler, Chem. Phys. Lett. 192, 1 (1992)

[56] S. Williams, D. S. Green, S. Sethuraman, and R. Zare, J. Am. Chem. Soc. 114, 9122 (1992)

[57] M. Versluis, G. Meijer, and D. W. Chandler, Appl. Optics 33 (15), 3289 (1994)

[58] Marc Bowden, in, Proceedings of the 7th international Symposium on

Laser-Aided Plasma Diagnostics, Fukuoka, Japan 1995

[59] Marc Bowden, private communication

[60] J.A. Gray, J.E.M. Goldsmith, and R. Trebino, Optics Letters, 18 (6), 444 (1993)

[61] J. P. Booth, M. Fadlallah, J. Derouard, and N. Sadeghi, Appl. Phys. Lett. 65, 819, (1994)

[62] U. Czarnetzki, D. Luggenhölscher, and H.F. Döbele, to be published 